\title{
Scaling law of diffusivity generated by a noisy telegraph signal with fractal intermittency
}

\author{
Paolo Paradisi ${ }^{1,2}$, Paolo Allegrini ${ }^{3}$ \\ ${ }^{1}$ Istituto di Scienza e Tecnologie dell'Informazione “A. Faedo” (ISTI-CNR), Via Moruzzi 1, 56124 Pisa, Italy \\ ${ }^{2}$ BCAM - Basque Center for Applied Mathematics, Alameda de Mazarredo, 14 E-48009 Bilbao, Basque Country - Spain \\ ${ }^{3}$ Scuola Superiore Sant'Anna, P.zza Martiri della Libertà 7, 56127 Pisa, Italy
}

\begin{abstract}
In many complex systems the non-linear cooperative dynamics determine the emergence of self-organized, metastable, structures that are associated with a birth-death process of cooperation. This is found to be described by a renewal point process, i.e., a sequence of crucial birth-death events corresponding to transitions among states that are faster that the typical long-life time of the metastable states. Metastable states are highly correlated, but the occurrence of crucial events is typically associated with a fast memory drop, which is the reason for the renewal condition. Consequently, these complex systems display a power-law decay and, thus, a long-range or scale-free behavior, in both time correlations and distribution of inter-event times, i.e., fractal intermittency.

The emergence of fractal intermittency is then a signature of complexity. However, the scaling features of complex systems are, in general, affected by the presence of added white or short-term noise. This has been found also for fractal intermittency.

In this work, after a brief review on metastability and noise in complex systems, we discuss the emerging paradigm of Temporal Complexity. Then, we propose a model of noisy fractal intermittency, where noise is interpreted as a renewal Poisson process with event rate $r_{p}$. We show that the presence of Poisson noise causes the emergence of a normal diffusion scaling in the long-time range of diffusion generated by a telegraph signal driven by noisy fractal intermittency. We analytically derive the scaling law of the long-time normal diffusivity coefficient. We find the surprising result that this long-time normal diffusivity depends not only on the Poisson event rate, but also on the parameters of the complex component of the signal: the power exponent $\mu$ of the inter-event time distribution, denoted as complexity index, and the time scale $T$ needed to reach the asymptotic power-law behavior marking the emergence of complexity. In particular, in the range $\mu<3$, we find the counter-intuitive result that normal diffusivity increases as the Poisson rate decreases.

Starting from the diffusivity scaling law here derived, we propose a novel scaling analysis of complex signals being able to estimate both the complexity index $\mu$ and the Poisson noise rate $r_{p}$.
\end{abstract}

Keywords: scaling, self-similarity, noise, time series analysis, signal processing, fractal intermittency, complex systems

\section{Introduction}

Complexity is nowadays observed in many scientific disciplines, from nanotechnologies and cell biology to human social activities. In all these fields, cooperative behaviors typically emerge from systems composed of many degrees of freedom, e.g., many individuals, functional units or nodes in a network. Cooperative behavior means a dynamical interaction among the system's units, which are coupled with strong non-linear interactions. In a complex system, such cooperative dynamics determine the occurrence of self-organized or coherent structures, which emerge at the global level or at some scale larger than the typical scales of the micro-dynamics.

\subsection{Metastability and Temporal Complexity: emergence of fractal intermittency}

In many complex systems such self-organized structures are not associated with a long-time equilibrium, but with metastability. Metastable states have a relatively long, but finite, lifetime. After this life-time, the self-organization is quenched by dynamical instabilities. Such instabilities increase slowly enough to maintain the self-organized structure almost stable for a long-time period, after which a fast acceleration towards an unstable region occurs. This is associated with a rapid chaotic transition to another state. The transition is typically characterized by low predictability and, then, by a fast drop in memory and self-organization. From a phenomenological point of view, the signature of this behavior is tracked in the abrupt and rapid changes of some signal experimentally observed in the complex system. In the following, these fast changes are denoted as crucial or complex events.

Brain dynamics is probably the most important example of a complex system and it is a prototype of this kind of behavior. In fact, metastability is a basic concept for the analysis of brain data and in the modeling of the brain information processing. It is nowadays well established that ElectroEncephaloGrams (EEG) display these rapid changes, which are called Rapid Transition Processes (RTPs) and mark the transition between two quasi-stationary periods in EEG traces [1, 2]. These abrupt changes can be characterized as steep variations in the signal or in its increments, corresponding to a discrete derivative, or as rapid changes in the topology of a network, where the 
topology could be evaluated, for example, as a functional connectivity (e.g., by means of thresholded covariance matrix). In the case of brain data, RTPs are a prototype of complex events. RTPs have been recognized to mark passages between successive neural metastable assemblies and are the signature of selforganization in the brain $[3,4]$. Neural assemblies are associated with transient information flow among different neurons with the goal of developing a specific brain function, such as the processing of information coming from an external stimulus and the response to it (e.g., Event Related Potentials).

A possible dynamical origin of metastability and of associated complex transition events is discussed in Refs. [5, 6, 7]. These authors propose a dynamical model for the brain information processing, but the paradigm of metastability introduced therein can be used also as a general paradigm for complex transition events. In fact, the authors model the brain dynamics by means of a stable heteroclinic channel. This is essentially a set of trajectories in the vicinity of a heteroclinic skeleton, consisting of saddle points and unstable separatrices. As known, this determine a slow motion towards the saddle points, which the authors interpret as metastable cognitive states preserving coherence or self-organization for a long time period. When the complex system approaches a saddle point, then a sudden acceleration occurs in which the system experiences a rapid motion towards another saddle point (i.e., another metastable cognitive state), whose neighbourhood is reached in a relatively short time. This rapid transition is associated with a low predictability and, then, with the drop of self-organization and memory with the consequence that the self-organized structure is annihilated and replaced by a new one.

These transitions are "rapid" or "fast" with respect to the long life-time of the self-organized states. As a consequence, the complexity of the system is, characterized by a birth-death process of self-organization. Considering also the fast memory drop, this process can be modelled through a renewal point process [8] (see also [9, 10] for a brief introduction to renewal processes). This stochastic process is a sequence of complex events occurring randomly in time. ${ }^{1}$ In the particular case of a pure renewal processes, inter-event times, or Waiting Times (WTs), are independent identically distributed (i.i.d.) random variables. This means that there's no statistical dependence among successive events and WTs. Then, the Probability Density Function (PDF) of the WTs, denoted as $\psi(\tau)$, can be computed as the histogram of the WTs without considering their chronological order $^{2}$. However, it is worth noting that the renewal property does not rule out the possibility of having strong memory in the time interval between two events. The slow power-law decay in the WT-PDF is associated with a relatively high statistical

\footnotetext{
${ }^{1}$ Theoretically, each event corresponds to a time instant, but in real data it has a finite duration time. However, this time scale is so short with respect to the long life-time of metastable states that it can be modeled as a time point event.

${ }^{2}$ We note that, when a renewal process is perturbed by an external signal, it looses its original renewal property. Then, a chronological order appears in the event sequence. Nevertheless, the renewal condition plays an important role in the theoretical modeling of these perturbed point processes, allowing to derive both analytical and numerical results. (see, e.g., [10, 11, 12, 13, 14, 15]).
}

weight of long WTs, so that the time-averaged computation results in long-range correlations also in time series with renewal events [16]. This is related to the time evolution of the rate of event production $r(t)$, which is essentially the mean number of events per time unit $[8,10,17]$. In general, the rate is not constant between two events, and it is renewed at its initial value in correspondence of each event occurrence. This rate is also denoted as age-specific failure rate in the Cox's book [8], where the term age is referred to the time change of the rate, which is measured from the occurrence of the last event.

It is easy to prove that the unique renewal process with constant rate $r(t)=r_{p}$ is the Poisson process, whose WT-PDF is an exponential function with decay rate $r_{p}: \psi(\tau)=\exp \left(-r_{p} \tau\right)$. The Poisson process describes the transition events in a timehomogeneous Markov chain, whose correlation is known to be given exactly by the same exponential form of the WT-PDF $\psi(\tau)$. Then, the Poisson process corresponds to Markovian dynamics, with exponential correlation function, and events occur due to a total randomness in the underlying dynamics. In more detail, the system's local units are independent from each other and, consequently, there's no emergence of self-organization. The events are simply related to the local dynamics and the global events come from a totally random superposition of independent events, which it is known to give rise to a Poisson distribution [8]. Consequently, the emergence of a Poisson sequence of events is associated with a lack of complexity ${ }^{3}$.

\subsection{Structural vs. Temporal Complexity: an intermittency- based measure of complexity}

At variance with Poisson systems, in a complex system the typical experimental observation is that of a WT-PDF displaying a inverse power-law in the long-time tail. The emergence of a renewal point process with power-law decay in the WT-PDF is a condition denoted as fractal intermittency [9, 18, 19, 20, 21], being the inverse power-law tail a signature of self-similarity in the long-time behavior of the WT-PDF. As said above, this renewal process with power-law intermittency is associated with the birth and death of self-organized metastable states. the power exponent can be used as a measure of the self-similarity scaling and it is denoted as complexity index and it is essentially based on the intermittency emerging in the time evolution of metastable structures.

There are several examples of complex systems displaying a fractal intermittent behavior: ecological systems [22], neural dynamics [23], blinking quantum dots [24, 25, 26], social dynamics [27], brain information processing [28, 29, 30, 16, 31, $32,18,19,33,1,3,4]$, atmospheric turbulence [34, 17, 35, 9], earthquakes [36], single particle tracking in cell biology [37], molecular biology [20].

This kind of complexity has been recently denoted as Temporal Complexity (TC) [38, 39] to distinguish it from the more extensively investigated Structural or Spatial Complexity, which

\footnotetext{
${ }^{3}$ We note that an observed Poisson behavior of intermittent events, even if plausible, could be sometimes erroneous, especially in presence of perturbing signals, and a genuine complexity could be hidden under the Poisson behavior (see, e.g., [14])
} 
is characterized through topological measures of network connectivity structures, such as the degree distribution [31] or the avalanche size distribution [40]. TC is essentially related to fractal intermittency and it has been evaluated through the complexity index. Theoretically, Temporal and Structural Complexity are different properties that can emerge indipendently from each other, and it is not clear if they are always linked or not. About this aspect, an interesting result was found for critical phenomena [41], which are known to display spatial complexity [31, 40]. A less known, but well-established finding is given in Ref. [42], where the authors proved that the fluctuations of a random field at the critical point, which is the order parameter, are described in terms of a Type-I intermittent dynamical map similar to the well-known Manneville map [43]. This is the same as fractal intermittency, essentially defining TC. Consequently, these authors proved that, in critical phenomena, spatial and temporal complexity are strictly connected. More recently, emergence of TC was found in some network models at the critical point, such as neural networks or a decision making model, which is basically a $2 \mathrm{D}$ version of the Ising model [38, 44, 45]. This is a strong indication that critical systems are also complex, not only in the topological sense, but also according to TC. An explicit analytical relationship between Temporal and Structural Complexity is given in the case of a random walker on a complex network [48]. Then, the estimation of the complexity index related to TC is also the signature of criticality and of spatial complexity.

Following the RTP concept and exploiting the associated algorithm for the processing of EEG signals [2], the evaluation of the complexity index in brain data can be found in Ref. [16], where a concept of global event has been used in order to characterize the overall brain complexity of healthy people. These findings are in agreement with other, better known, complexity measures, such as the long-range (power-law) correlations in both time and space [16]. The complexity index was not estimated directly from the WT-PDF, but through a different approach, which will be denoted here as Event-Driven Diffusion Scaling (EDDiS). For the sake of clarity, this is briefly described in Appendix A. In synthesis, the main idea is to use a random walk to study long range correlations. This idea, dating back to the works of Hurst, was made popular in the nineties by Peng et al. [49]. The EDDiS method studies both the longrange correlation index through second-moment analysis (Hurst index $H$ ) and the rescaling properties of the resulting diffusion (scaling index $\delta$ ) [50]. The relations between $H$ and $\delta$ depend on the particular process under study: For example, they coincide in the case of fractional Brownian motion and differ in the case of Lévy diffusion [51]. Among the many studies adopting diffusion to detect anomalies, it is worth mentioning the work of Scafetta et al., where the two indexes were confronted to check for hidden renewals [52]. The EDDiS method rests on this idea, with the adoption of more walking rules. Details can be found in Refs. [16, 9] and references therein. Most of the analytical results this method is based on were originally reported either in [46] or in [47]. In Appendix A we adopt the terminology introduced in the work of Raffaelli et al. [47]. EDDiS approach was used because the direct estimation of the complexity in- dex from the WT-PDF is not reliable, as it was shown in Ref. [53]. For intermittent systems, this is related to the presence of secondary events, which can be considered as a sort of noisy sequence added to that of genuine complex events. The WTPDF is affected by the blurring effect of noisy secondary events and it is often difficult to derive the complexity index from the WT-PDF. Even more, the added noise can also determine the emergence of an effective complexity index that is completely different from the real one [53].

On the contrary, it has been proved that the EDDiS approach is able to separate different regimes of both diffusion scalings $\delta$ and $H$, but only for the Asymmetric Jump (AJ) and Symmetric Jump (SJ) walking rules (see Appendix Appendix A) $[15,17,9]$. This is a crucial property when noise affects the recorded time series. In general the power exponent of the WT-PDF is different from the one of the underlying complexity when detected events are not renewal, as in the case of superposition of independent processes [53]. This discrepancy also occurs when renewal events trigger or deterministic or quasideterministic pseudo-events [54]. Also in these cases the indirect method, similar to the EDDiS approach, is cabable of revealing the underlying renewal complexity [54].

\subsection{Noise in complex systems}

Noise has different roles in complexity. Here, the term noise denotes random fluctuations with rapidly decaying memory and spatial coherence and, then, with short-term and spatially local correlations. This means that the dynamics of the noise is approximately Markovian, or even without any memory, and consequently, it is not a direct manifestation of self-organization. Even though, noise can play a fundamental role in complex systems and, in some cases, can also trigger the emergence of complexity itself (see, e.g., [45]).

However, the presence of noise, especially if the consequence of interactions with the external environment, could be a disturbance to the clear emergence of complex behavior. Following this interpretation, the role of added noise in complex systems has been extensively investigated by many authors, as it can affect the observed scaling properties $[55,53,15,56,57$, $58,59,60]$. In particular, many authors have studied the effect of added noise on monofractal and multifractal signals. In Ref. [56], the authors applied Detrended Fluctuation Analysis (DFA), in the multifractal version (MF-DFA) to a mono-fractal signal with an additive white noise and found that the analysis shows a spurious multi-fractality. In particular, they found an underestimation in the generalized Hurst exponents $h(q)$ and pronounced crossovers in the generalized fluctuation functions $F_{q}(s)$ at positions decreasing with the increase of the moment order $q .{ }^{4}$ In order to solve the discrepancy between simulated and real data, the authors of Ref. [57] compared two different

\footnotetext{
${ }^{4}$ These authors investigated also the effect of periodicities, such as seasonal trends. In renewal processes, the effect of external periodic perturbations was also extensively studied in Refs. [11, 13, 10, 14]. This aspect also deserves some care in time series analysis, as it affects the observed correlation properties of the signal $[56,11]$.
} 
approaches to study the predictability of extreme events in simulated multifractal records with added white noise. With this approach, these authors could explain some findings in physiological and financial records.

Further, the presence of short-term noise can affect the calculation of long-range correlations [55, 58] and of intermittency features [53, 15]. Recently, the presence of added Gaussian noise, white or exponentially correlated, has been recognized to play a crucial role for the interpretation of single particle tracking of biomolecules in the cell [59].

\subsection{Poisson noise in fractal intermittency}

As said above, both $\mathrm{AJ}$ and $\mathrm{SJ}$ rules are able to separate different regimes in the diffusion scaling. A common assumption for these different regimes is that of a multi-fractal or multiscaling signal. However, an alternative hypothesis is that the emergence of different diffusion scalings is related to contributions coming from different kind of events in the intermittent time series. Actually, two contribution are sufficient to explain a mistaken multi-scaling behavior: a genuine complex sequence superposed to secondary events related to short-time effects. In particular, the presence of a normal scaling in the short-time range of the $\mathrm{AJ}$ diffusion process mark the presence of noisy secondary events $[16,17]$. It is well known that the normal scaling corresponds to a complexity index $\mu>3$ (see Appendix A), which is always a power-law decay, but this range of $\mu$ is considered outside the long-range regime, as it admits a finite correlation time for both stationary and aged correlation functions [61].

When noisy secondary events are present in the time series, the emergence of normal scaling $(H=\delta=0.5)$ in the shorttime range for the $\mathrm{AJ}$ and $\mathrm{SJ}$ rules is not surprising as second moments and statistical distributions are, in this case, linearly superposed. More surprisingly, normal scaling emerge also in the Symmetric Velocity (SV) rule, which is dichotomous telegraph signal (see Appendix A), but this normal scaling is seen in the long-time range $[16,15]$. This is related to a non-linear superposition of effects and, for this reason, the estimation of the diffusion scaling is often not reliable for the SV rule.

In this work, we give a theoretical explanation for the emergence of the long-time normal scaling in the SV rule Following Ref. [53], we can assume that noisy secondary events are generated by a Poisson process. In the following, this component of the signal will be denoted as Poisson noise. This Poisson noise is superposed to the genuine complex signal, which is given by a sequence of complex events with fractal intermittency. The two components are independent from each other and each one is a renewal process by itself ${ }^{5}$. In Section 2 we recall the derivation of the effective WT-PDF $\psi(\tau)$ of the point process

\footnotetext{
${ }^{5}$ In the case of the superposition of independent renewal processes, the total rate of the global point process is given by the sum of the single rates [8]. Further, when the single renewal process are all Poisson processes, the resulting process is again a renewal Poisson process whose total event rate is given by the sum of the single rates. Surprisingly, when at least a single renewal process $N_{1}(t)$ is Non-Poisson, the renewal condition is no more garanteed for the global process. This is due to the dependence of the rate on the aging time, i.e., the
}

resulting from the superposition of a complex signal with the Poisson noise [53, 15]. In Section 3 we derive the asymptotic regime of normal scaling for the diffusion generated by the telegraph signal, i.e., the SV walking rule, associated with the noisy fractal intermittency. In particular, an analytical expression for the long-time diffusivity is derived in the limit of small Poisson event rate. Based on the results of Section 3, a scaling analysis characterizing the complexity of intermittent signals with added noise is proposed in Section 4. Finally, in Section 5 some conclusions are drawn.

\section{Fractal intermittency with Poisson noise: effective WT-PDF}

A point process is defined as a sequence of events occurring randomly in time, whose main feature is the probability distribution of inter-event times $\psi(\tau)$. The WTs can be correlated or statistically independent and, in the last case, the process is renewal and the events are mutually independent. For a Poisson process, the WT-PDF:

$$
\psi_{p}(\tau)=r_{p} \exp \left(-r_{p} \tau\right)
$$

The parameter $r_{p}$ is the Poisson rate of event production, i.e., the averaged expected number of events per time unit and is given by the inverse of the average time:

$$
r_{p}=\frac{1}{\left\langle\tau_{p}\right\rangle} ; \quad\left\langle\tau_{p}\right\rangle=\int_{0}^{\infty} \tau \psi_{p}(\tau) d \tau .
$$

A sequence of events can follow from the superposition of two or more point processes. A superposition of independent Poisson renewal processes is again a Poisson process, whose global rate is the sum of rates of the single Poisson processes [8].

As anticipated in the Introduction, we assume that the experimental sequence of events is given by the superposition of two independent sequences: the first is generated by a Poisson process and the second one by a Non-Poisson process, where the first one models the Poisson noise component and the second one is the genuine complex signal. This model, originally denoted as Copy and Mistake Map (CMM) and proposed for modeling DNA sequences in Ref. [63] and in linguistics [48], is here denotes as Temporal Mixed Model (TMM). In Fig. 2 a sketch of a possible sequence coming out from the superposition of two point process is displayed. The two series of events are represented by the associated sequence of occurrence times: $\left\{t_{i}^{(p)}\right\}$ and $\left\{t_{i}^{(n)}\right\}, i=1,2, \ldots$, for the Poisson and Non-Poisson processes, respectively. Both processes are assumed to be renewal processes homogeneous in time, i.e., with both WT-PDFs not

time passed from the last event of that Non-Poisson single process. In fact, let us assume that an event of another renewal process $N_{2}(t)$ occurs after that of the Non-Poisson process. Independently from assuming the $N_{2}(t)$ to be Poisson or not, the occurrence of this event reset the aging time and the rate of $N_{2}(t)$, but not the rate of $N_{1}(t)$. Consequently, the total rate has a different shape after this event and, then, the process is not renewal, because the rate is not always reset to its initial value. 


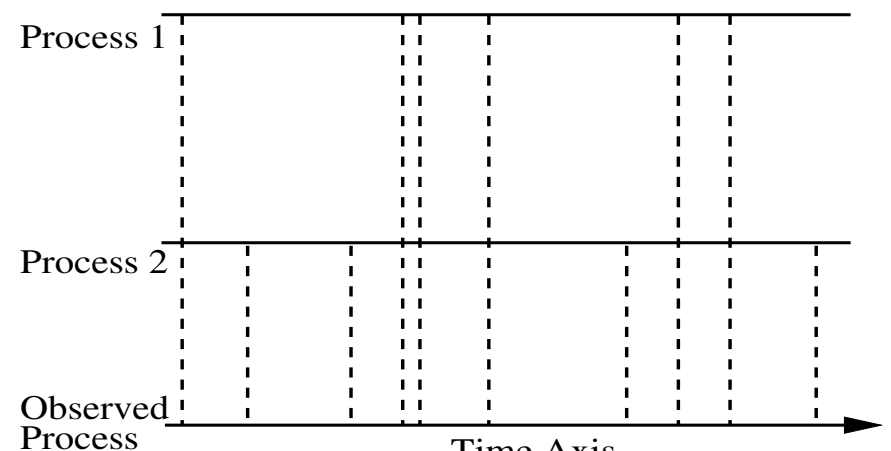

Time Axis

Figure 1: Sketch of the superposition of two point processes. Note that the the observer is not able to distinguish a priori the two hidden processes and the two kinds of events.

changing in time. The inter-event times or Waiting Times (WT) are given by:

$$
\tau_{i}^{(p)}=t_{i}^{(p)}-t_{i-1}^{(p)} ; \quad \tau_{i}^{(n)}=t_{i}^{(n)}-t_{i-1}^{(n)} ; \quad i=1,2, \ldots .
$$

The WT-PDF of the Poisson process is exactly the exponential distribution $\psi_{p}(\tau)$ of Eq. (1), while the WT-PDF of the Non-Poisson process $\psi_{n}(\tau)$ is non-exponential. Here we focus on an inverse power-law asymptotic behavior in the WT-PDF: $\psi(\tau) \sim 1 / \tau^{\mu}$. A simple prototype of this behavior is given by the distribution:

$$
\psi_{n}(\tau)=(\mu-1) \frac{T^{\mu-1}}{(T+\tau)^{\mu}}
$$

where the multiplicative factor is due to the normalization condition. This Non-Poisson process with power-law decay of WT$\mathrm{PDF}$ is a prototype of a complex system generating renewal events. The time scale $T$ is the time needed to reach the asymptotic inverse power-law behavior and is here denoted as complexity emergence time. The complexity index $\mu$ is the main feature of fractal intermittency generated by the system's dynamics. The average time is given by:

$$
\left\langle\tau_{n}\right\rangle=\left\{\begin{array}{cc}
\infty & \text { if } \mu \leq 2 \\
\frac{T}{\mu-2} & \text { if } \mu>2
\end{array}\right.
$$

It is interesting to note that, even if the two processes are renewal, the resulting global point process is no more renewal in general. The only exception is the sum of Poisson renewal processes [8]. This is easily seen by introducing the Cox's agespecific rate of event production, defined as the (conditional) probability density that an event occurs in an infinitesimal time interval $[t, t+d t]$, given that no events occurred in the time interval $\left[t_{i}, t\right]$, being $t_{i}$ the occurrence time of the last observed event. This is proved to be given by [8]:

$$
r(t)=\frac{\psi(t)}{\Psi(t)}=-\frac{\dot{\Psi}(t)}{\Psi(t)}
$$

where

$$
\Psi(t)=\int_{t}^{\infty} \psi\left(t^{\prime}\right) d t^{\prime}
$$

is the Survival Probability Function of the WTs (WT-SPF). The Poisson rate $r_{p}$ is constant in time, but the rate $r_{n}(t)$ of a NonPoisson process is, in general, a decreasing function in the time interval between two events. In a renewal process, the rate restarts always from the initial value $r(0)$ in correspondence of an event. In the superposition of two renewal processes, the global rate is the sum of the two single rates. It is easy to see that, in the global sequence of superposed events, the global rate $r(t)$ restarts from the value $r(0)=r_{p}+r_{n}(0)$ in correspondence of a Non-Poisson event. On the contrary, when a Poisson event occurs, the Non-Poisson rate does not restarts from the resetting value $r_{n}(0)$ and the value of the global rate is given by $r\left(t_{*}\right)=r_{p}+r_{n}\left(t_{*}\right)$, where $t_{*}$ is the time since the last NonPoisson event. It is worth noting that, in an experimental event sequence, the time $t_{*}$ is not directly observed.

Even if, in the TMM, the total sequence of event is not renewal, it is possible to derive an analytical expression for the global $\psi(\tau)$, independently from the particular shape of $\psi_{n}(\tau)$, but assuming that both processes are stationary. This is done as follows.

The occurrence of an event can be divided into four complementary situations, depending on the fact that the initial and the final instants of the inter-event time interval can be either a Poisson or a non-Poisson event. By denoting with $n(t)(p(t))$ the presence of a non-Poisson (Poisson) event at time $t$, we have:

(i) the occurrence of a Non-Poisson event after a NonPoisson event $(n(t), n(t+\tau))$;

(ii) the occurrence of a Poisson event after a Non-Poisson event (n,p) $(n(t), p(t+\tau))$;

(iii) the occurrence of a Non-Poisson event after a Poisson event $(\mathrm{p}, \mathrm{n})(p(t), n(t+\tau))$;

(iv) the occurrence of a Poisson event after a Poisson event $(\mathrm{p}, \mathrm{p})(p(t), p(t+\tau))$.

Being these four situations a complete set of non-intesecting subsets of the probability space, it is possibile to apply the summation of the single probabilities:

$$
\begin{gathered}
\psi(\tau)=\langle P[n(t), n(t+\tau)]+P[n(t), p(t+\tau)] \\
+P[p(t), n(t+\tau)]+P[p(t), p(t+\tau)]\rangle_{t},
\end{gathered}
$$

where $P[a, b]$ is the joint probability density for both event $a$ and event $b$ and $\tau$ the Waiting Time (WT) between the two events. The time-average $\langle\ldots\rangle$ is needed as $\psi(\tau)$ is the effective WT-PDF, experimentally estimated from the entire sequence, and not a probability conditioned from the particular time instant. This is often the only reliable WT-PDF that can be estimated from an experimental time series, but it is also the most useful property in the stationary case. The stationarity assumption allows us to compute the probabilities $\langle P[a, b]\rangle_{t}$ in Eq. (8) by using the time-average probability for an event to belong to the Poisson, $P_{p}$, or to the Non-Poisson sequences, $P_{n}$. As an example, the first term in the r.h.s of $(8), P[n(t), n(t+\tau)]$, is the joint probability of first having a Non-Poisson event $\left(P_{n}\right)$, then a Non-Poisson event after a time $\tau$ and no Poisson events in between, which becomes

$$
\langle P[n(t), n(t+\tau)]\rangle_{t}=P_{n} \Psi_{p}(\tau) \psi_{n}(\tau)=P_{n} e^{-r_{p} \tau} \psi_{n}(\tau),
$$


The stationarity assumption is encoded in the coefficient $P_{n}$. We have also used the fact that the Poisson process has a constant rate and, consequently, no aging [61, 25, 62].

In order to evaluate $P_{p}$ and $P_{n}$ it is enough to observe that the probabilities $P_{p}$ and $P_{n}$ are inversely proportional to the respective average times:

$$
P_{n} \propto \frac{1}{\left\langle\tau_{n}\right\rangle} ; \quad P_{p} \propto \frac{1}{\left\langle\tau_{p}\right\rangle} ; \quad\left\langle\tau_{p, n}\right\rangle=\int_{0}^{\infty} \tau \psi_{p, n}(\tau) d \tau
$$

Note that, being $\tau_{n}=\infty$ for $\mu \leq 2,\left\langle\tau_{n}\right\rangle$ can be defined only in the range $\mu>2$, so that our calculation applies only in this range of $\mu$. This also corresponds to the fact that, in a Gibbs ensemble of independent realizations of the renewal process, all prepared in the same way at some initial instant $t_{0}$, the stationary regime is not possible for $\mu<2$ because the time needed to relax the initial condition is infinite. After normalization,

$$
P_{n}=\frac{\left\langle\tau_{p}\right\rangle}{\left\langle\tau_{p}\right\rangle+\left\langle\tau_{n}\right\rangle} ; \quad P_{p}=\frac{\left\langle\tau_{n}\right\rangle}{\left\langle\tau_{p}\right\rangle+\left\langle\tau_{n}\right\rangle} ; \quad P_{n}+P_{p}=1
$$

As above, the property of Poisson processes, i.e., the constant rate, can be used to compute $P[n(t), p(t+\tau)]$. This is the joint probability of first having a Non-Poisson event $\left(P_{n}\right)$, then a Poisson event after a time $\tau$ and no Non-Poisson events in between, which becomes

$$
P[n(t), p(t+\tau)]=P_{n} \Psi_{n}(\tau) r e^{-r_{p} \tau} .
$$

As above, the property of Poisson processes, i.e., the constant rate, was used. Next, we could use the equality $P[p(t), n(t+$ $\tau)]=P[n(t), p(t+\tau)]$, a consequence of the time-reversal symmetry of both processes and, consequently, also of the global process. We prove this fact by deriving $P[p(t), n(t+\tau)]$ in a direct way, namely by writing

$$
P[p(t), n(t+\tau)]=P_{p} e^{-r_{p} \tau} \psi_{n}^{\infty}(\tau),
$$

where $\psi_{n}^{\infty}(\tau)$ is the infinitely-aged waiting time distribution [61]. In the right-hand side of this expression the first factor denotes the probability of starting from a Poisson event, the second one denotes the fact that no Poisson events occur, while the third term, in line with the other cases, is the probability density of a Non-Poisson event occurring after a waiting time $\tau$. Notice that we have lost track of the previous Non-Poisson event, and this forces us to use an aged $\psi$. Due to stationarity assumption, this term is given by the infinitely-aged one. In detail [61],

$$
\begin{array}{r}
\psi_{n}^{t_{a}}(\tau)=\int_{-t_{a}}^{0} R\left(t^{\prime}+t_{a}\right) \psi_{n}\left(\tau-t^{\prime}\right) d t^{\prime}, \text { where } \\
R(t) \equiv \sum_{i=0}^{\infty} \psi_{n}^{i}(t), \quad \psi_{n}^{i}(t)=\int_{0}^{\infty} d t^{\prime} \psi_{n}^{i-1}\left(t-t^{\prime}\right) \psi_{n}\left(t^{\prime}\right),
\end{array}
$$

$\psi_{n}^{0}(t)=\delta(t)$, a Dirac $\delta$. For $t_{a}=\infty, R(\infty)=1 /\left\langle\tau_{n}\right\rangle[61]$, so that

$$
\psi_{n}^{\infty}(\tau)=\frac{1}{\left\langle\tau_{n}\right\rangle} \int_{-\infty}^{0} \psi_{n}\left(\tau-t^{\prime}\right) d t^{\prime}=\frac{\Psi_{n}(\tau)}{\left\langle\tau_{n}\right\rangle} .
$$

Substituting this relation and the expression for $P_{p}$ given in Eq. (11) into Eq. (13), we get:

$$
\begin{aligned}
& P[p(t), n(t+\tau)]=\frac{\left\langle\tau_{n}\right\rangle}{\left\langle\tau_{p}\right\rangle+\left\langle\tau_{n}\right\rangle} \frac{\Psi_{n}(\tau)}{\left\langle\tau_{n}\right\rangle} e^{-r_{p} t}= \\
& =\frac{\left\langle\tau_{p}\right\rangle}{\left\langle\tau_{p}\right\rangle+\left\langle\tau_{n}\right\rangle} \Psi_{n}(\tau) r e^{-r_{p} t}=P_{n} \Psi_{n}(\tau) r e^{-r_{p} \tau},
\end{aligned}
$$

identical to $P[n(t), p(t+\tau)]$, where we used Eq. (11) for $P_{n}$ and $r=1 /\left\langle\tau_{p}\right\rangle$. Finally, the probability of having a Poisson event $\left(P_{p}\right)$, and then a Poisson event after a time $\tau$ with no nonPoisson events in between is

$$
P[p(t), p(t+\tau)]=P_{p} r e^{-r_{p} \tau} \Psi_{n}^{\infty}(\tau)
$$

where, again, the aged $\Psi_{n}^{\infty}$ has to be used. Inserting (9),(12),(13),(16) and (17) into (8) yields

$$
\psi(\tau)=\left\{P_{n}\left[\psi_{n}(\tau)+2 r \Psi_{n}(\tau)\right]+P_{p} r \Psi_{n}^{\infty}(\tau)\right\} e^{-r_{p} \tau}
$$

and the survival probability $\Psi(t) \equiv \int_{t}^{\infty} \psi(\tau) d \tau$ can be calculated by direct integration. The result is

$$
\Psi(\tau)=\left\{P_{n} \Psi_{n}(\tau)+P_{p} \Psi_{n}^{\infty}(\tau)\right\} e^{-r_{p} \tau} .
$$

Notice that before the exponential cutoff in (18) we have the joint action of two inverse-power laws, with indexes $\mu$ and $\mu-1$, being $\psi_{n}(\tau)$ given by Eq. (4) and:

$$
\psi_{n}^{\infty}(\tau)=(\mu-2) \frac{T^{\mu-2}}{(T+\tau)^{\mu-1}} .
$$

The same observation applies to the SPF $\Psi(\tau)$ of Eq. (19), but with indexes $\mu-1$ and $\mu-2$, being:

$$
\Psi_{n}(\tau)=\left(\frac{T}{T+\tau}\right)^{\mu-1} ; \quad \Psi_{n}^{\infty}(\tau)=\left(\frac{T}{T+\tau}\right)^{\mu-2} .
$$

If $r$ were vanishingly small, the asymptotic behavior of $\psi(\tau)$ and $\Psi(\tau)$ would be dictated by the index $\mu$ and $\mu-1$, respectively. We recall that the theory rests on the case $\mu>2$.

In Ref. [53] it was shown that the fit of EEG data with the WT-PDF of TMM, given in Eq. 18, is more reliable of the pure inverse power-law fit. Further, the estimated value of $\mu$ is in agreement with the EDDiS analysis $(\mu>2)$. On the contrary, a power-law fit gives an erroneous value $\mu<2$. Further, WT-SPF was also shown to mimick a strecthed exponential decay, which is probably due to the superposition of two inverse power-law decays.

\section{Diffusivity scaling law of the noisy telegraph signal}

The telegraph signal $\xi(t)$, or SV rule, is built starting from the sequence of events as explained in Appendix A. It can take alternative values \pm 1 , with sign selection, according to a coin-tossing procedure, in correspondence of event occurrence times. In absence of Poisson-like noisy events, or for low rates of the Poisson noise, the SV rule is accurate as the other walking rules. However, as already said in the Introduction, the SV rule is often not reliable due to the presence of spurious Poisson-like events in the time series, which determine the emergence of a 
long-time normal diffusion scaling $H_{\mathrm{sv}}=\delta_{\mathrm{sv}}=0.5$. One of the reason for this collapse is the vanishing of the anomalous ballistic peaks. The ballistic peaks, corresponding to walkers never changing their velocity are asymptotically proportional to the velocity autocorrelation function [70] and, in the absence of noise, are responsible for the difference between $\delta$ and $H$, as the diffusion process becomes bi-fractal [71], the two dimensions corresponding to $\delta$ (scaling of the central body, highlighted by small moments of the diffusion) and $H$ (driven by the ballistic peaks, highlighted by large moments). In general the central body is built up according to the convolution of the "flights" (distributed as $\psi$ ), while the ballistic peaks follow, as mentioned, the autocorrelation function. When noise is superimposed both functions decay exponentially, so the body becomes Gaussian and the peaks tend to vanish. This leads to $\delta=H=0.5$, i.e. standard diffusion.

From the SV walking rule given in Appendix A, the fluctuating velocity can be written in the following way:

$$
\frac{d X(t)}{d t}=\xi(t)= \pm 1 ; \quad t_{i}<t<t_{i+1}
$$

and, integrating with respect to time as in Eq. (A.1), we get:

$$
X(t)= \pm\left(t-t_{i}\right)+X\left(t_{i}\right) \text { for } t \in\left[t_{i}, t_{i+1}\right]
$$

where $t_{i}$ is the occurrence time of the $\mathrm{i}$-th event.

From Eq. (A.1) it is possible to derive the following expression for the second moment under a stationarity assumption [64]:

$$
\sigma^{2}(t)=\left\langle(X(t)-X(0))^{2}\right\rangle=2\left\langle\xi^{2}\right\rangle \int_{0}^{t} d t^{\prime} \int_{0}^{t^{\prime}} d t^{\prime \prime} \Phi_{\xi}\left(t^{\prime \prime}\right),
$$

where $\Phi_{\xi}\left(t^{\prime \prime}\right)$ is the stationary auto-correlation function of the signal $\xi(t)$. For a point process with WT-PDF $\psi(\tau)$ and WT-SPF $\Psi(\tau)$ it is possible to apply the Geisel-Zacherl-Radons formula [65]:

$$
\Phi_{\xi}(t)=\frac{1}{\langle\tau\rangle} \int_{t}^{\infty} d t^{\prime}\left(t^{\prime}-t\right) \psi\left(t^{\prime}\right)=\frac{1}{\langle\tau\rangle} \int_{t}^{\infty} d t^{\prime} \Psi\left(t^{\prime}\right)
$$

Subtituting Eq. (25) into Eq. (24), the variance can be computed by knowing the WT-SPF of the process:

$$
\sigma^{2}(t)=\frac{2\left\langle\xi^{2}\right\rangle}{\langle\tau\rangle} \int_{0}^{t} d t^{\prime} \int_{0}^{t^{\prime}} d t^{\prime \prime} \int_{t^{\prime \prime}}^{\infty} d t^{\prime \prime \prime} \Psi\left(t^{\prime \prime \prime}\right)
$$

Being the Geisel's formula, Eq. (25), independent from the renewal assumption, the same applies for Eq. (26), which is the mathematical expression for the second moment. Consequently, an important aspect of this analytical derivation is that the scaling $H$ does not depend on the renewal property, but only on the WT-PDF $\psi(\tau)$.

From the direct evalutation of the second moment by means of Detrended Fluctuation Analysis (DFA) [49] it was found a normal diffusion scaling in the long-time range:

$$
\left\langle(X(t)-X(0))^{2}\right\rangle \sim 2 D t .
$$

This can be proved considering the exponential cutoff in the WT-SPF given in Eq. (19), which determines a finite correlation time for the signal $\xi(t)$. The emergence of a normal diffusion scaling in the long-time limit allows to define a long-time diffusivity coefficient:

$$
D=\lim _{t \rightarrow \infty} \frac{1}{2} \frac{d\left\langle(X(t)-X(0))^{2}\right\rangle}{d t},
$$

which is well-defined for times longer than the correlation time, i.e., the time scale characterizing the transition to the normal scaling.

Applying Eq. (26) to the definition of diffusivity $D$, it results:

$$
D=\frac{\left\langle\xi^{2}\right\rangle}{\langle\tau\rangle} \int_{0}^{\infty} d t \int_{t}^{\infty} d t^{\prime} \Psi\left(t^{\prime}\right) .
$$

Substituting Eqs. (19) and (21) in the previous one and applying the substitutions $y=r_{p} t$ and $z=r_{p} t^{\prime}$ in the integrals:

$$
\begin{aligned}
& D\left(\mu, r_{p}, T\right)=D_{p}+D_{n} \\
& D_{i}=\frac{P_{i}\left\langle\xi^{2}\right\rangle}{\langle\tau\rangle} I_{\alpha_{i}} \quad ; \quad i=p, n \\
& I_{\alpha_{i}}\left(r_{p}, T\right)=\frac{1}{r_{p}^{2}} \int_{0}^{\infty} d y \int_{y}^{\infty} d z \frac{\exp (-z)}{\left(1+z /\left(r_{p} T\right)\right)^{\alpha_{i}}} \\
& \alpha_{p}=\mu-2 \quad ; \quad \alpha_{n}=\mu-1 .
\end{aligned}
$$

Now, it is possible to prove the following crucial result:

$$
r_{p} T<<1 \Rightarrow I_{\alpha} \sim r_{p}^{\alpha-2} T^{\alpha}
$$

This can be proved in two different ways.

\section{First Proof}

The integral $I_{\alpha}$ in Eq. (32) can be splitted into two contributions: 5

$$
\begin{aligned}
I_{\alpha}= & \frac{1}{r^{2}} \int_{0}^{r T} d y \int_{y}^{\infty} d z \frac{\exp (-z)}{(1+z /(r T))^{\alpha}}+ \\
& +\frac{1}{r^{2}} \int_{r T}^{\infty} d y \int_{y}^{\infty} d z \frac{\exp (-z)}{(1+z /(r T))^{\alpha}} .
\end{aligned}
$$

Under the assumption $r T<<1$, the first contribution is negligible. In the second term, it is possible to neglect 1 with respect to $z /(r T)$ in the denominator, being $z /(r T)>y /(r T)>1$. Further, the lower limit $y=r T$ can be approximated with 0 , so that it results:

$$
I_{\alpha} \simeq C_{\alpha} r_{p}^{\alpha-2} T^{\alpha} \quad ; \quad C_{\alpha}=\int_{0}^{\infty} d y \int_{y}^{\infty} d z \frac{\exp (-z)}{z^{\alpha}} .
$$

This proves the relationship given in Eq. (34).

\section{Second Proof}

In Eq. (32) the order of the integrals can be exchanged by taking some care in rewriting the integration domain:

$$
\begin{aligned}
I_{\alpha} & =\frac{1}{r_{p}^{2}} \int_{0}^{\infty} d z \int_{0}^{z} d y \frac{\exp (-z)}{\left(1+z /\left(r_{p} T\right)\right)^{\alpha}}= \\
& =\frac{1}{r_{p}^{2}} \int_{0}^{\infty} d z \frac{z \exp (-z)}{\left(1+z /\left(r_{p} T\right)\right)^{\alpha}},
\end{aligned}
$$


and substituting $z=r_{p} T x$ in the integral:

$$
I_{\alpha}=T^{2} \int_{0}^{\infty} d x \frac{x e^{-\left(r_{p} T\right) x}}{(1+x)^{\alpha}}=-T^{2} \frac{d}{d\left(r_{p} T\right)} \int_{0}^{\infty} d x \frac{e^{-\left(r_{p} T\right) x}}{(1+x)^{\alpha}}
$$

This expression is in the form of a Laplace transform with Laplace variable $u=r_{p} T$, so that a Tauberian theorem can be applied [66]:

$$
\begin{aligned}
f(x) & \sim \frac{1}{x^{\alpha}}(x \rightarrow+\infty) \Leftrightarrow \\
& \Leftrightarrow \bar{f}(u) \sim 1-A u^{\alpha-1} \quad\left(u=r_{p} T \rightarrow 0\right),
\end{aligned}
$$

where $f(x)=(1+x)^{-\alpha}$. This results in the following asymptotic expression:

$$
\begin{aligned}
I_{\alpha}\left(r_{p}, T\right) & =-T^{2} \frac{d}{d r_{p}}\left(1-A\left(r_{p} T\right)^{\alpha-1}\right)= \\
& =\alpha A T^{2}\left(r_{p} T\right)^{\alpha-2} \propto r_{p}^{\alpha-2} T^{\alpha}
\end{aligned}
$$

then again proving Eq. (34).

Now, substituting Eq. (35) into Eqs. (30-30) and considering that $\left\langle\xi^{2}\right\rangle=1$ for a dichotomous signal:

$$
\begin{aligned}
D\left(\mu, r_{p}, T\right) & =\frac{P_{p}}{\langle\tau\rangle} C_{\alpha_{p}} r_{p}^{\alpha_{p}-2} T^{\alpha_{p}}+\frac{P_{n}}{\langle\tau\rangle} C_{\alpha_{n}} r_{p}^{\alpha_{n}-2} T^{\alpha_{n}}= \\
& =\frac{P_{p}}{\langle\tau\rangle} C_{\mu-2} r_{p}^{\mu-4} T^{\mu-2}+\frac{P_{n}}{\langle\tau\rangle} C_{\mu-1} r_{p}^{\mu-3} T^{\mu-1},
\end{aligned}
$$

where also Eq. (33) for $\alpha_{p}$ and $\alpha_{n}$ is used. Note that $\langle\tau\rangle$ is the average waiting time of the entire sequence and $1 /\langle\tau\rangle$ is the global rate (i.e., total number of events per time unit). The total number of events is clearly the sum of the number of events of each single process (Poisson and Non-Poisson), i.e., it is the sum of the two single average rates:

$$
\frac{1}{\langle\tau\rangle}=\frac{1}{\tau_{p}}+\frac{1}{\tau_{n}}=r_{p}+\frac{\mu-2}{T} .
$$

Finally, substituting this expression and Eq. (11) into Eq. (38), it results the following asymptotic expression for the diffusivity coefficient:

$$
D\left(\mu, r_{p}, T\right)=A r_{p}^{\mu-3} T^{\mu-2} ; \quad r_{p} T \ll 1 ; \quad \mu>2
$$

with

$$
A=C_{\mu-2}+(\mu-2) C_{\mu-1} .
$$

Eq. (40) is a power-law relationship giving the scaling law of the long-time diffusivity coefficient with respect to the Poisson noise rate $r_{p}$ and the time scale $T$ of the complex component, with power exponents dependent on the complexity index $\mu$.

Finally, it is worth noting that the regime $r_{p} T \ll 1$ is the same as $\tau_{p} \gg T$. Considering Eq. (5) for $\mu>2$, we get $\tau_{n} \ll \tau_{p} /(\mu-2)$. Apparently, for fixed average times $\tau_{n}$ and $\tau_{p}$, this condition is more easily fulfilled when $\mu$ approaches 2 . However, in this limit, a constant $\tau_{n}$ is obtained if $T \rightarrow 0^{+}$as $\mu \rightarrow 2^{+}$, which corresponds to a Dirac delta function for the WT-PDF and to a step function in 0 for the WT-SPF, which does not seem a realistic approximation. This means that the time scale $T$ of Non-Poisson complex events is more important than the average time $\tau_{n}$.

Fig. 2 illustrates a numerical validation of Eq. (40). We generated TMM sequences with varying poisson rates $r$ superimposed to a non-Poisson distribution obeying Eq. (4) with $\mu=2.3$ and $T=1.0$ (arbitary time units). The total duration of each sequences was 100 millions of units. We see that the diffusion coefficients rescale according to $r$ with the predicted behavior, highlighted by the dashed curve.

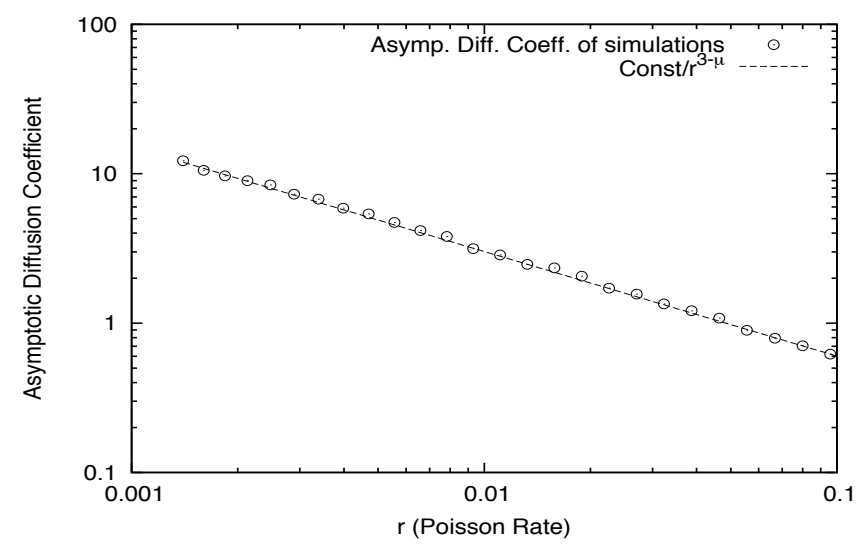

Figure 2: Asymptotic diffusion coefficients of TMM $(\mu=2.3, T=1.0)$ as a function of supermposed Poisson rate.

\section{Proposal for a novel scaling analysis of complex signals}

The diffusivity scaling law, Eq. (40), can be exploited to develop a statistical algorithm of time series analysis. In particular, let us assume that a time series is characterized by particular time point events that can be detected with some event detection algorithm. In the case of a complex system, the resulting event sequence is expected to display Temporal Complexity in the form of a renewal point process with fractal intermittency. However, due to the reasons explained above, noisy secondary events are typically present in the time series. The secondary events are generated by the superposition of independent sources, which are typically not a part of the cooperative dynamics determining the emergence of the self-organized structures. Then, the temporal structure of the secondary events can be treated as a Poisson noise with constant rate $r_{p}$.

It is also known that the superposition of two independent renewal Poisson processes with rate $r_{1}$ and $r_{2}$ is again a renewal Poisson process with total rate $r_{T}=r_{1}+r_{2}$. This important property can be exploited in the following way:

(1) Given the experimental sequence of event occurrence times $\left\{t_{i}\right\}, i=1,2,3, \ldots$, let us evaluate the total average time $\langle\tau\rangle$. This is clearly given by $T_{e} / N_{e}$, being the $T_{e}$ the total duration time of the experimental time series and $N_{e}$ the total number of observed events.

Further, the EDDiS method can be applied to get the diffusion scalings $H$ and $\delta$, different estimates of the complexity index $\mu$ and, if possible, to verify the renewal condition. 
(2) Let us generate $N$ random sequences of Poisson events $\left\{t_{j}^{\prime}\right\}_{k}, j=1,2,3, \ldots$ with the constrain that the occurrence time of the last event must be less than the total duration time $T_{e}$. Each sequence has a different Poisson rate $r_{k}, k=1, \ldots, N$.

(3) Then, for each $k$, let us build sequences that are given by the superposition of the experimental sequence $\left\{t_{i}\right\}$ and of the artificial Poisson sequence with rate $r_{k}:\left\{t_{j}^{\prime}\right\}_{k}$.

(4) The artificial Poisson sequence, when it is added to the experimental sequence, behaves exactly as explained in Sections 2 and 3. Thus, it is possible to sum the different rates and, if a real Poisson noise with rate $r_{p}$ is present, then the new Poisson rate is given by the sum of the real rate $r_{p}$ and of the artificially generated rate $r_{k}$. Consequently, the diffusivity scaling law, Eq. (44), can be rewritten in the following way:

$$
\tilde{D}\left(r_{k}\right)=D\left(\mu, r_{p}+r_{k}, T\right)=B(T)\left(r_{p}+r_{k}\right)^{\mu-3} ; \mu>2
$$

where

$$
B(T)=A T^{\mu-2}
$$

and $A$ is the constant given in Eq. (41). The function $\tilde{D}\left(r_{k}\right)$ has been introduced to underline the functional dependence of the effective diffusivity $D$ on the set of rates $r_{k}$, which is a set of known parameters of the analysis.

(5) For each $k$, it is possible to apply the SV rule on the generated sequence of events (real+artificial Poisson) and the DFA can be applied on the generated diffusion process to get a numerical estimate $D_{k}$ of the long-time diffusivity coefficient $D\left(r_{k}\right)=D\left(\mu, r_{p}+r_{k}, T\right)$.

(6) Finally, a set of couples $\left(D_{k}, r_{k}\right)$ is obtained, and these couples can be plotted, thus giving a discrete version of the function $\tilde{D}\left(r_{k}\right)$ given in Eq. (42). A best fit procedure with the function $\tilde{D}(r)$, Eqs. (42-43), with respect to the three parameters $\mu, r_{p}$ and $T$ can be performed.

It is also possible to apply a best fit with respect to only two parameters: $r_{p}$ and $T$. In this case, $\mu$ is evaluated from the EDDiS method.

It is important to stress that the estimation of the complexity index $\mu$, the Poisson rate $r$ and of the complexity emergence time $T$, being based on the SV rule, does not depend on the renewal assumption, but only on the functional shape of $\psi(\tau)$. This means that the method is only based on the existence of a sequence of crucial events, i.e., a point process, without any need of the renewal assumption. Even though, our proposal, based on the diffusivity scaling law given in Eq. (40), can be exploited not only to estimate $\mu, r_{p}$ and $T$, but also to check if the complex events are in agreement with the renewal assumption. This can be done coupling this analysis with the EDDiS approch and compare the estimated values of $\mu$.

\section{Discussions}

Eq. (40) is the main result of the paper. This formula relates the long-time diffusivity, generated by the integration of the telegraph signal, to the rate of the Poisson noise $r_{p}$ and, surprisingly, also to the parameters of the complex component of the signal, i.e., the time scale $T$ to reach the asymptotic powerlaw, self-similar, regime, and the complexity index $\mu$, which characterizes the system's self-similarity.

The power-law dependence of long-time diffusivity coefficient $D$ on the Poisson rate $r_{p}$ is a counter-intuitive result. A linear relationship would be expected, but the mathematical calculations show that the dependence of diffusivity $D$ on the Poisson rate $r_{p}$ is mediated by the complex component of the signal, thus giving the following power exponent:

$$
\beta=\mu-3 .
$$

Let us also note that $\beta<0$ when $\mu<3$, so that, in presence of complexity, the diffusivity coefficient increases as the Poisson rate decreases, which is again a counter-intuitive result.

This result was obtained considering the TMM, a point process consisting of two independent renewal processes. The first is a renewal point process with fractal intermittency, which is a prototype of Temporal Complexity [38, 39]. The second one is a Poisson noise, i.e., a sequence of events with Poisson statistics, whish is superposed to the complex component. In analogy with the concept of white noise added to monoor multi-fractal signals, which was discussed by many authors $[55,56,57,58,59,60]$, the Poisson noise can encode the effects of the external environment, or it can represent the source of errors in a statistical algorithm for data analysis, or it can be even a sort of residual of the cooperative dynamics, which, in this case, would generate both self-organization and uncorrelated noise. It is not always clear a priori if the noise component in a complex signal is due to the external environment or a sort of residual manifestation of non-linear interactions, which determine also the emergence of cooperative behavior. Linear superposition and independence is ensured in the first case. In the second case, it is difficult to understand if the two components (noisy and complex) are linearly superposed, if they are independent from each other and which are their contributions to total energy and total entropy of the signal. In this paper we did not discuss deeply the origin of noise, which need further investigations, and we followed essentially the approach of other authors by considering a linear superposition of noisy and complex signals. Even in the second case, it is reasonable to assume a linear superposition when the source of noise comes from dynamical scales much faster and smaller than the temporal and spatial scales of the cooperative components of the non-linear dynamics, i.e., those giving rise to metastable selforganized structures.

Interestingly, the TMM includes not only the complexity index $\mu$ and the Poisson noise rate $r_{p}$, but also a time scale $T$. As said in Section 2, $T$ is the time needed to display a clear complexity behavior, i.e., the time above which the inter-event times are distributed according to a inverse power-law function 
with a well-defined exponent $\mu$. This time scale is not a secondary parameter. On the contrary, it could play a crucial role in the characterization of complexity emergence in cooperative systems. For example, in the TMM, this is underlined by the relationship $r_{p} T \ll 1$, which is the basic condition necessary to derive the scaling law for the long-time diffusivity, as given in Eq.(40).

\section{Appendix A. Analysis of Event-Driven Diffusion Scaling}

The EDDiS method as described herein was applied for the first time in Ref. [16] to evaluate the complexity index of brain EEG data in the basal, or resting state, condition.

The basic ingredient of EDDis method is the generation of three different diffusion processes that are driven by the same experimental sequence of events and, then, the evaluation of two different scaling exponents. In the case of renewal events, the relatiohships among the different scaling exponents and of these exponents with the complexity index are known. Then, it is possible to derive independent estimations of the complexity index associated with events driving all the diffusion processes. When the evaluations of the complexity index, obtained through these different scaling analyses applied to different diffusion processes, are compatible with each other, the renewal condition is reasonably proved and, further, a robust estimation of the complexity index is obtained.

Let us assume that the event sequence is the manifestation of Temporal Complexity, which is described through a (longrange) power-law decay in the WT-PDF and by a complexity index $\mu<3: \psi(\tau) \sim 1 / \tau^{\mu}$. Then, given the sequence of events, the three different diffusion process are generated by means of three walking rules ${ }^{6}$. The walkers are allowed to move only in correspondence of an event occurrence, thus generating a event-driven diffusion process. In general, starting from the same event sequence, different walking rules can give different scaling properties. Given the sequence of event occurrence times $t_{0}, t_{1}, t_{2}, \ldots$ corresponding to the events $0,1,2, \ldots$, we have the following walking rules:

(a) Asymmetric Jump (AJ) rule:

the walker makes a positive jump $\left(\xi\left(t_{n}\right)=1\right)$ in correspondence of each event $n$, otherwise it stands $(\xi(t)=0)$. In other words, $\xi(t)$ is a sequence of pulses of constant intensity.

(b) Symmetric Jump (SJ) rule: as in the AJ rule, but the walker can make positive or negative jumps in correspondence of an event: $\xi\left(t_{n}\right)= \pm 1$. The sign \pm is chosen with a coin tossing prescription.

(c) Symmetric Velocity (SV) rule:

the walker moves with constant velocity towards a given direction, in the time interval between two events, then

${ }^{6}$ These walking rules, and the associated diffusion processes, are essentially particular cases of a more general model known as Continuous Time Random Walk, which was extensively studied by the sixties $[67,68]$. a new random direction (positive or negative velocity) is chosen in correspondence of an event:

$\xi(t)= \pm 1 ; t_{n}<t \leq t_{n+1}$. As in the $\mathrm{SJ}$ rule, the sign is chosen with a coin tossing prescription.

Each random walk is then defined through a signal $\xi(t)$, which is a kind of random discontinuous velocity, taking two or three different discrete values (two for AJ and SV and three for SJ). The diffusion variable of the random walk is computed by simply integrating the signal $\xi(t)$ :

$$
X(t)=X_{0}+\int_{0}^{t} \xi\left(t^{\prime}\right) d t^{\prime}
$$

In the $\mathrm{AJ}$ and $\mathrm{SJ}$ rules the diffusion variable $X(t)$ is a piecewise constant function with finite discontinuities in correspondence of the events, and the integral in Eq. (A.1) can be reduced to a discrete sum. On the contrary, in the SV rule, $X(t)$ is a increasing or decreasing function depending on the sign of the velocity $\xi . X(t)$ is a continuous function, but with discontinuous derivative in correspondence of the events.

The scaling properties of these random walks were extensively investigated in several papers (see [16, 9, 47, 72] and references therein). In particular, two scaling indices were found in the asymptotic long-time limit: the self-similarity index $\delta$ of the diffusion probability distribution, defined by:

$$
P(x, t)=\frac{1}{t^{\delta}} F\left(\frac{x}{t^{\delta}}\right)
$$

and the scaling exponent $H$ of the second moment:

$$
\sigma^{2}(t)=\left\langle(X(t)-\bar{X})^{2}\right\rangle \sim t^{2 H},
$$

where $\bar{X}$ is the mean value of $X(t)$. These scaling exponents have been computed in the case of renewal power-law events, i.e., fractal intermittency, with complexity index, $\mu$. The ana-

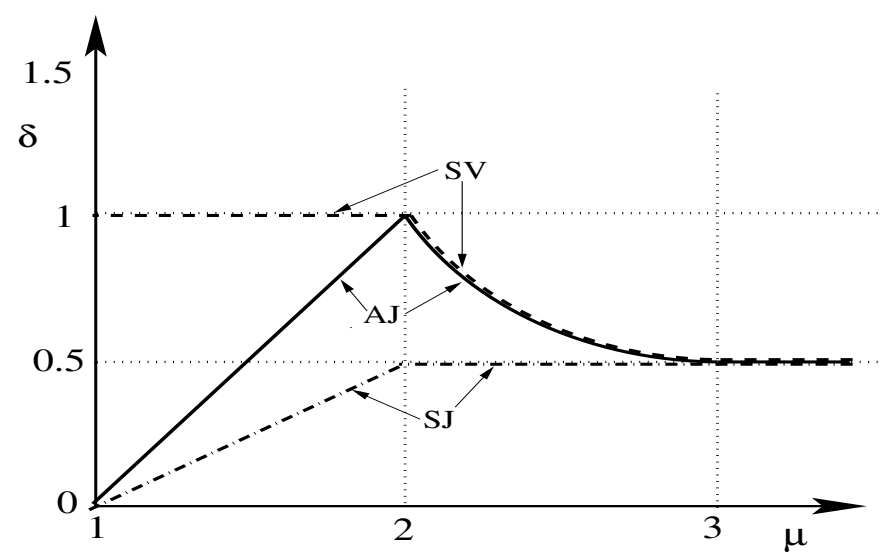

Figure A.3: Scaling $\delta$ vs. complexity index $\mu$ for the three walking rules: AJ (continuous line), SJ (dotted-dashed line) and SV (dashed line).

lytical expressions for the diffusion scaling exponents $\delta$ and $H$ as a function of the complexity index $\mu$ are plotted in Figures A.3-A.4 and summarized in the following: 


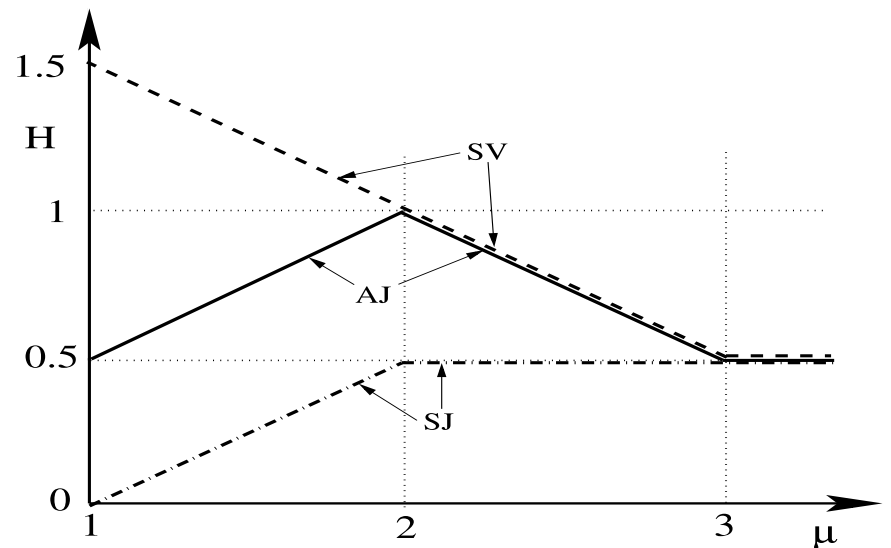

Figure A.4: Scaling $H$ vs. complexity index $\mu$ for the three walking rules: AJ (continuous line), SJ (dotted-dashed line) and SV (dashed line).

$$
\begin{aligned}
& \delta_{A J}(\mu)= \begin{cases}\mu-1 ; & 1<\mu<2 \\
1 /(\mu-1) ; & 2 \leq \mu<3 \\
1 / 2 ; & \mu \geq 3\end{cases} \\
& H_{A J}(\mu)= \begin{cases}\mu / 2 ; & 1<\mu<2 \\
2-\mu / 2 ; & 2 \leq \mu<3 \\
1 / 2 ; & \mu \geq 3\end{cases} \\
& \delta_{S V}(\mu)= \begin{cases}1 ; & 1<\mu<2 \\
1 /(\mu-1) ; & 2 \leq \mu<3 \\
1 / 2 ; & \mu \geq 3\end{cases} \\
& H_{S V}(\mu)=\left\{\begin{array}{ll}
2-\mu / 2 ; & 1<\mu<3 \\
1 / 2 ; & \mu \geq 3
\end{array} \quad \mu \geq 2\right.
\end{aligned}
$$

The three rules give a normal scaling $\delta=H=1 / 2$ for $\mu \geq 3$, corresponding to normal (Gaussian) diffusion. This is a consequence of the generalized limit theorem for Lévy stable distribution [69]. For the SJ rule this is true also in the range $2<\mu \leq 3$, while AJ and SV rules are super-diffusive $(H>1 / 2)$ in all the interval $1<\mu<3$. On the contrary, the SJ rule is subdiffusive $(H<1 / 2)$ for $1<\mu<2$. Regarding the AJ rule in the range $1<\mu<2$, it is interesting to observe that, while being super-diffusive $(H>1 / 2)$, the scaling $\delta$ becomes less than $1 / 2$ for $\mu<3 / 2$. For pure Poisson events, the values of $\delta$ and $H$ are again $1 / 2$ and, in the long-time, we get a Gaussian diffusion. This is not surprising as the Poisson process corresponds to the limit $\mu \rightarrow \infty$.

Finally, it is worth noting that, when the values of $\mu$ evaluated using the two scaling analyses, DE for $\delta$ and DFA for $H$, are in agreement with each other, then the EDDis approach can also give a reasonable justification of the renewal assumption [52, 9].

\section{Author contribution}

Both authors developed and discussed the main idea of the paper. They developed both the EDDiS method for the analysis of complex time series and carried out the calculation of the effective WT distribution of noisy fractal intermittency. Both authors have set the calculation of the diffusivity scaling law.

P.P. carried out the mathematical passages to derive the diffusivity scaling law, Eq. (40). The paper was written by P.P. with some insights from P.A.

[1] A. A. Fingelkurts, A. A. Fingelkurts, Brain-Mind Operational Architectonics Imaging: Technical and Methodological Aspects. Open Neuroimag. J. 2 (2008) 73-93.

[2] A. Y. Kaplan, A. A. Fingelkurts, A. A. Fingelkurts, B. S. Borisov, B. S. Darkhovsky, Nonstationary nature of the brain activity as revealed by EEG/EMG: methodological, practical and conceptual challenges. Signal Process. 85 (2005) 2190-2212.

[3] A. A. Fingelkurts, A. A. Fingelkurts, C. F. H. Neves, Natural world physical, brain operational, and mind phenomenal space-time. Phys. Life Rev. 7 (2010) 195-249.

[4] A. A. Fingelkurts, A. A. Fingelkurts, C. F. H. Neves, Consciousness as a phenomenon in the operational architectonics of brain organization: Criticality and self-organization considerations. Chaos, Solitons and Fractals 55 (2013) 13-31.

[5] M. I. Rabinovich, R. Huerta, V. S. Afraimovich, Dynamics of Sequential Decision Making. Phys. Rev. Lett. 97 (2006) 188103.

[6] M. I. Rabinovich, R. Huerta, P. Varona, V. S. Afraimovich, Transient Cognitive Dynamics, Metastability, and Decision Making. PLoS Comput. Biol. 4(5) (2008) e1000072.

[7] M.I. Rabinovich, V.S. Afraimovich, C. Bick, P. Varona, Information flow dynamics in the brain. Phys. Life Rev. 9 (2012) 51-73.

[8] D. R. Cox, Renewal Theory, Methuen \& Co. Ltd., London, 1962.

[9] P. Paradisi, R. Cesari, A. Donateo, D. Contini, P. Allegrini, Diffusion scaling in event-driven random walks: an application to turbulence. Rep. Math. Phys. 70 (2012) 205-220.

[10] P. Paradisi, R. Cesari, P. Grigolini, Superstatistics and renewal critical events. Cent. Eur. J. Phys. 7 (2009) 421-431.

[11] O.C. Akin, P. Paradisi, P. Grigolini, Periodic trend and fluctuations: The case of strong correlation. Physica A 371 (2006) 157-170.

[12] P. Paradisi, P. Grigolini, S. Bianco, O. C. Akin, Renewal aging in nonhomogeneous Poisson processes with periodic rate modulation. Int. J. Bif. Chaos 18 (2008) 2681-2691.

[13] S. Bianco, P. Grigolini, P. Paradisi, A fluctuating environment as a source of periodic modulation. Chem. Phys. Lett. 438 (2007) 336-340.

[14] O. C. Akin, P. Paradisi, P. Grigolini, Perturbation-induced emergence of Poisson-like behavior in non-Poisson systems. J. Stat. Mech.: Theory Exp., (2009) P01013, doi: 10.1088/1742-5468/2009/01/P01013 .

[15] P. Allegrini, P. Paradisi, D. Menicucci, R. Bedini, A. Gemignani, L. Fronzoni, Noisy cooperative intermittent processes: From blinking quantum dots to human consciousness. J. Phys.: Conf. Series 306 (2011) 012027.

[16] P. Allegrini, D. Menicucci, R. Bedini, L. Fronzoni, A. Gemignani, P. Grigolini, B.J. West, P. Paradisi, Spontaneous brain activity as a source of ideal 1/f noise. Phys. Rev. E 80 (2009) 061914.

[17] P. Paradisi, R. Cesari, A. Donateo, D. Contini, P. Allegrini, Scaling laws of diffusion and time intermittency generated by coherent structures in atmospheric turbulence. Nonlinear Processes in Geophysics 19 (2012) 113126; P. Paradisi et al., Corrigendum, Nonlinear Processes in Geophysics 19 (2012) 685.

[18] P. Paradisi, P. Allegrini, A. Gemignani, M. Laurino, D. Menicucci, A. Piarulli, Scaling and intermittency of brain events as a manifestation of consciousness. AIP Conf. Proc. 1510 (2013) 151-161.

[19] P. Allegrini, P. Paradisi, D. Menicucci, M. Laurino, R. Bedini, A. Piarulli, A. Gemignani, Sleep unconsciousness and breakdown of serial critical intermittency: New vistas on the global workspace. Chaos, Solitons and Fractals 55 (2013) 32-43.

[20] P. Paradisi, D. Chiarugi, P. Allegrini, A renewal model for the emergence of anomalous solute crowding in liposomes. BMC Systems Biology, 9 (2015) S7.

[21] P. Paradisi, Fractional calculus in statistical physics: The case of time 
fractional diffusion equation. Comm. Appl. Industr. Math., to appear (2015).

[22] R. V. Solé, J. Bascompte: Self-organization in Complex Ecosystems. Princeton University Press, Princeton (2006).

[23] W. Gerstner and W. M. Kistler: Spiking Neuron Models, Cambridge University Press, Cambridge (2002).

[24] P. Paradisi, P. Allegrini, F. Barbi, S. Bianco, P. Grigolini, Renewal, modulation and blinking quantum dots. AIP Conf. Proc. 800 (2005) 92-97.

[25] S. Bianco, P. Grigolini, P. Paradisi, Fluorescence intermittency in blinking quantum dots: renewal of modulation? J. Chem. Phys. 123 (2005) 174704.

[26] F.D. Stefani, J.P. Hoogenboom, E. Barkai, Beyond quantum jumps: Blinking nanoscale light emitters. Physics Today 62 (2009) 34-39.

[27] T. Maillart, D. Sornette, S. Frei, T. Duebendorfer, A. Saichev, Phys. Rev. E 83 (2011) 056101.

[28] J. M. Beggs, D. Plenz, Neuronal Avalanches in Neocortical Circuits. J. Neurosci. 23 (2003) 11167-11177.

[29] D. Plenz, T. C. Thiagarjan, The organizing principle of neuronal avalanches: cell assemblies in the cortex? Trends Neurosci. 30, (2007) 101-110.

[30] S. Laureys and G. Tononi (Eds.): The neurology of consciousness, Elsevier, Amsterdam (2009).

[31] D. R. Chialvo, Emergent complex neural dynamics. Nat. Phys. 6 (2010) 744-750; D. Fraiman, P. Balenzuela, J. Foss, D.R. Chialvo, Ising-like dynamics in large-scale functional brain networks. Phys. Rev. E 79 (2009) 061922

[32] P. Allegrini, P. Paradisi, D. Menicucci, A. Gemignani, Fractal complexity in spontaneous EEG metastable state transitions: new vistas on integrated neural activity. Frontiers in Physiology 1, 128 (2010).

[33] P. Allegrini, P. Paradisi, D. Menicucci, A. Gemignani, Is temporal scaling at the basis of allometry? Comment on "Physiologic time: A hypothesis" by West and West. Phys. Life Rev. 10 (2013) 233-234.

[34] P. Paradisi, R. Cesari, D. Contini, A. Donateo, L. Palatella, Characterizing memory in atmospheric time series: an alternative approach based on renewal theory. Eur. Phys. J. Special Topics 174 (2009) 207-218.

[35] P. Paradisi, R. Cesari, F. Mainardi, A. Maurizi and F. Tampieri: A generalized Fick's law to describe non-local transport effects. Phys. Chem. Earth 26(4) (2001) 275-279.

[36] A. Saichev, D. Sornette, "Universal" Distribution of Inter-earthquake Times Explained. Phys. Rev. Lett. 97 (2006) 078501.

[37] I. Y. Wong, et al., Anomalous Diffusion Probes Microstructure Dynamics of Entangled F-Actin Networks. Phys. Rev. Lett. 92 (2004) 178101; A. V. Weigel, B. Simon, M. M. Tamkun, D. Krapf, Ergodic and nonergodic processes coexist in the plasma membrane as observed by single-molecule tracking. Proc. Nat. Acad. Sci. USA 108 (2011) 6438-6443; J. H. Jeon, et al., In Vivo Anomalous Diffusion and Weak Ergodicity Breaking of Lipid Granules. Phys. Rev. Lett. 106 (2011) 048103; R. Metzler, J. H. Jeon, A. G. Cherstvy, E. Barkai, Anomalous diffusion models and their properties: non-stationarity, non-ergodicity, and ageing at the centenary of single particle tracking. Phys. Chem. Chem. Phys. 16 (2014) 24128-24164

[38] M. Turalska, B. J. West, P. Grigolini, Temporal complexity of the order parameter at the phase transition. Phys. Rev. E 83 (2011) 061142.

[39] J. U. Mafahim, D. Lambert, M. Zare, P. Grigolini, Complexity matching in neural networks. New J. Phys. 17 (2015) 015003.

[40] F. Lombardi, H. J. Herrmann, C. Perrone-Capano, D. Plenz, L. de Arcangelis, Balance between Excitation and Inhibition Controls the Temporal Organization of Neuronal Avalanches. Phys. Rev. Lett. 108 (2012) 228703

[41] D. Sornette, Critical phenomena in natural sciences. Springer-Verlag, Berlin (2006)

[42] Y. F. Contoyiannis, F. K. Diakonos, Criticality and intermittency in the order parameter space. Phys. Lett. A 268 (2000) 286; Y. F. Contoyiannis, F. K. Diakonos, A. Malakis, On the intermittent dynamics of critical fluctuations. Phys. Rev. Lett. 89 (2002) 035701.

[43] P. Manneville, Intermittency, self-similarity and 1/f Spectrum in dissipative dynamical systems. Journal de Physique (France) 41 (1980) 12351243

[44] N. W. Hollingshad, M. Turalska, P. Allegrini, B. J. West, P. Grigolini, A new measure of network efficiency. Physica A 391 (2012) 1894-1899.

[45] E. Lovecchio, P. Allegrini, E. Geneston, B. J. West, P. Grigolini, From Self-Organized to Extended Criticality. Front. Physiol. 3 (2012) 98 doi: 10.3389/fphys.2012.00098.

[46] S. B. Lowen, M. C. Teich MC, Fractal renewal processes generate 1/f noise. Phys Rev E 47 (1993) 9921001.

[47] P. Grigolini, L. Palatella, G. Raffaelli, Asymmetric anomalous diffusion: an efficient way to detect memory in time series. Fractals 9 (2001) 439449

[48] P. Allegrini, P. Grigolini, L. Palatella, Intermittency and scale-free networks: a dynamical model for human language complexity. Chaos, Solitons and Fractals 20 (2004) 95-105.

[49] C. -K. Peng, S. V. Buldyrev, S. Havlin, M. Simons, H. E. Stanley, A. L. Goldberger, Mosaic organization of DNA nucleotides. Physical Review E 49 (1994) 1685

[50] N. Scafetta, P. Hamilton, P. Grigolini, The thermodynamics of social processes: the teen birth phenomenon. Fractals 9 (2001) 193-208.

[51] A. Montagnini, P. Allegrini, S. Chillemi, A. Di Garbo, P. Grigolini, Rescaling prescriptions: on the conflict between Hurt's analysis and the second moment prediction. Physics Letters A 244 (1998) 237-244.

[52] scafetta N. Scafetta, P. Grigolini, Scaling detection in time series: diffusion entropy analysis. Physical Review E 66 (2002) 036130.

[53] P. Allegrini, D. Menicucci, R. Bedini, A. Gemignani, P. Paradisi, Complex intermittency blurred by noise: Theory and application to neural dynamics. Phys. Rev. E 82 (2010) 015103.

[54] P. Allegrini, P. Grigolini, P. Hamilton, L. Palatella, G. Raffaelli, Memory beyond memory in heart beating, a sign of a healthy physiological condition. Physical Review E 65 (2002) 041926; P. Allegrini, R. Balocchi, S. Chillemi, P. Grigolini, P. Hamilton, R. Maestri, et al., Long-and shorttime analysis of heartbeat sequences: Correlation with mortality risk in congestive heart failure patients. Physical Review E 67 (2003) 062901; M. S. Mega, P. Allegrini, P. Grigolini, V. Latora, L. Palatella, A. Rapisarda, S. Vinciguerra, Power-law time distribution of large earthquakes. Physical Review Letters 90 (2003) 188501.

[55] J. W. Kantelhardt et al., Breathing during REM and non-REM sleep: correlated versus uncorrelated behaviour. Physica A 319 (2003) 447-457.

[56] J. Ludescher, M. I. Bogachev, J. W. Kantelhardt, A. Y. Schumann A. Bunde, On spurious and corrupted multifractality: The effects of additive noise, short-term memory and periodic trends. Physica A 390 (2011) 2480-2490.

[57] M. I. Bogachev, A. Bunde, On the predictability of extreme events in records with linear and nonlinear long-range memory: Efficiency and noise robustness. Physica A 390 (2011) 2240-2250.

[58] D. Rybski, S. V. Buldyrev, S. Havlin, F. Liljeros, H. A. Makse, Communication activity in a social network: relation between long-term correlations and inter-event clustering. Scientific Reports 2 (2012) 560.

[59] J. H. Jeon, E. Barkai, R. Metzler, Noisy continuous time random walks. J. Chem. Phys. 139 (2013) 121916.

[60] P. Pramukkul, A. Svenkeson, P. Grigolini, Effect of noise and detector sensitivity on a dynamical process: Inverse power law and Mittag-Leffler interevent time survival probabilities. Phys. Rev. E 89 (2014) 022107.

[61] P. Allegrini, et al., Correlation function and generalized master equation of arbitrary age. Phys. Rev. E 71 (2005) 066109.

[62] P. Allegrini, F. Barbi, P. Grigolini, P. Paradisi, Aging and renewal events in sporadically modulated systems. Chaos, Solitons and Fractals 34 (2007) 11-18.

[63] P. Allegrini, P. Grigolini, B. J. West, A dynamical approach to DNA sequences. Physics Letters A 211 (1996) 217.

[64] G. I. Taylor, Diffusion by continuous movements. Proc. Lond. Math. Soc. Series 2 (1921) 196.

[65] T. Geisel, A. Zacherl, G. Radons, Generic 1/f Noise in Chaotic Hamiltonian Dynamics Phys. Rev. Lett. 59 (1987) 2503.

[66] W. Feller, An introduction to probability theory and its applications, $2^{\text {nd }}$ edition, vol. II. Wiley, New York (1971).

[67] E. W. Montroll, Random walks on lattices. Proc. Symp. Appl. Math., Am. Math. Soc. 16 (1964) 193-220.

[68] G. H. Weiss, R. J. Rubin, Random Walks: Theory and Selected Applications. Adv. Chem. Phys. 52 (1983) 363-505.

[69] V. M. Zolotarev, One-dimensional Stable Distributions, American Mathematical Society, Providence (1983); P. Lévy: Theorie de l'Addition des Variables Aleatoires, Gauthier-Villars, Paris (1937).

[70] P. Allegrini, P. Grigolini, B. J. West, Dynamical approach to Levy processes. Physical Review E 54 (1996) 4760; M. Stefancich, P. Allegrini, L. Bonci, P. Grigolini, B. J. West, Anomalous diffusion and ballistic peaks: 
A quantum perspective. Physical Review E 57 (1998) 6625.

[71] P. Allegrini, J. Bellazzini, G. Bramanti, M. Ignaccolo, P. Grigolini, J. Yang, Scaling breakdown: a signature of aging. Physical Review E 66 (2002) 015101(R).

[72] L. Palatella, J. Perelló, M. Montero, J. Masoliver, Activity autocorrelation in financial markets. The European Physical Journal B-Condensed Matter and Complex Systems, 38 (2004) 671-677. 